\section{Bundesbeauftragter für den Datenschutz - Wechsel im Amt}

\section{Peter Schaar ist ausgeschieden}

Am 16. Dezember 2013 endete die 10jährige Amtsperiode von Peter Schaar. Er nahm das Amt des Bundesbeauftragten für den Datenschutz seit dem 17. Dezember 2003 wahr. Er wurde am 26. November 2008 vom Deutschen Bundestag wiedergewählt.

In seine Amtszeit fielen wichtige datenschutzpolitische Ereignisse, u. a. die Schaffung einer neuen Sicherheitsarchitektur in Deutschland nach den Terroranschlägen des 11. September 2001, verbunden mit der Erweiterung von Eingriffsbefugnissen für Polizei und Nachrichtendienste, die Diskussion über die Einführung der Vorratsdatenspeicherung, die Verbesserungen im Datenschutz im Zuge der im Jahr 2008 aufgedeckten Missstände beim Handel mit personenbezogenen Daten und dem Umgang mit Mitarbeiterdaten, die Diskussion über die Anpassung des Datenschutzrechts an die Herausforderungen des digitalen Zeitalters, die in die Beratungen der Vorschläge der EU-Kommission für eine Modernisierung des europäischen Datenschutzrechts mündeten sowie zuletzt die Enthüllungen zur massenhaften Überwachung der Kommunikation durch in- und ausländische Sicherheitsbehörden und die Debatte über die daraus zu ziehenden Konsequenzen.

\section{Andrea Voßhoff übernimmt}

Die Mitglieder des Deutschen Bundestags haben Frau Andrea Voßhoff am 19. Dezember 2013 zur neuen Bundesbeauftragten für den Datenschutz und die Informationsfreiheit gewählt.

Die 55-jährige Juristin Voßhoff erhielt in offener Wahl mit Stimmkarte und Wahlausweis 403 von 587 abgegebenen Stimmen. Mit Nein votierten 151 Abgeordnete, 31 enthielten sich. Es gab zwei ungültige Stimmen.

Das Amt des Bundesbeauftragten für den Datenschutz wurde 1978 eingerichtet und zum 1. Januar 2006 um das Amt des Bundesbeauftragten für die Informationsfreiheit ergänzt. In der Dienststelle arbeiten 85 Beschäftigte in Bonn und Berlin. Die Bundesbeauftragte berät und kontrolliert Bundesbehörden, andere öffentliche Stellen des Bundes sowie Telekommunikations- und Postdienstunternehmen. Zudem berät und kontrolliert sie die Durchführung von Sicherheitsüberprüfungen nach dem Sicherheitsüberprüfungsgesetz des Bundes, auch soweit sie private Unternehmen betreffen.

Die Bundesdatenschutzbeauftragte ist Mitglied der Konferenz der Datenschutzbeauftragten des Bundes und der Länder und vertritt Deutschland in der Artikel-29-Gruppe, einer europäischen Arbeitsgruppe der Datenschutzbeauftragten der EU-Mitgliedstaaten, sowie in den europäischen und internationalen Konferenzen der Datenschutz- und Informationsfreiheitsbeauftragten. Ferner wirkt sie in den Gemeinsamen Datenschutz-Kontrollgremien für Europol und das Schengener Informationssystem mit. Das Amt des Bundesbeauftragten für den Datenschutz wurde 1978 eingerichtet und zum 1. Januar 2006 um das Amt des Bundesbeauftragten für die In- formationsfreiheit ergänzt. In der Dienststelle arbeiten 85 Beschäftigte in Bonn und Berlin. Die Bundesbeauftragte berät und kontrolliert Bundesbehörden, andere öffentliche Stellen des Bundes sowie Telekommunikations- und Postdienstunternehmen. Zudem berät und kontrolliert sie die Durchführung von Sicherheitsüberprüfungen nach dem Sicherheitsüberprüfungsgesetz des Bundes, auch soweit sie private Unternehmen betreffen.

\section{Sächsischer DSB: 16. TB für den öffentlichen sowie 6. TB für den nicht-öffentlichen Bereich}

Der Sächsische Datenschutzbeauftragte, Andreas Schurig, stellte am 16.12.2013 die Tätigkeitsberichte für den öffentlichen und den nicht-öffentlichen Bereich vor. Die Berichtszeiträume beginnen im Jahr 2011 und enden im Jahr 2013. Die Berichte enthalten Beiträge zu Prüfvorgängen bei der sächsischen Verwaltung und bei sächsischen Unternehmen, zu Rechtsprechung und Rechtsetzung und zu Datensicherheitsfragen.

\section{Technische und gesellschaftliche Entwicklung}

\section{Der Überwachungsskandal}

Kurz nach Ende des Berichtszeitraums wurde durch die Veröffentlichungen von Edward Snowden ein Überwachungsskandal bisher unbekannten Ausmaßes durch Geheimdienste bekannt. Diese aktuelle Entwicklung kann wegen ihrer Bedeutung nicht ausgelassen bleiben. Und sie ist auch für den Freistaat Sachsen von Gewicht.

Mit der Begründung Terrorismusabwehr sammeln die Nachrichtendienste auf allen Ebenen personenbezogene Daten aus Kommunikationsvorgängen und werten diese aus. So sollen - so eine der letzten undementierten Meldungen - alleine die Bewegungsdaten von ca. 100 Millionen Handys weltweit einer permanenten Kontrolle durch die US-amerikanischen Nachrichtendienste unterliegen.

Es ist - natürlich - davon auszugehen, dass auch sächsische Entscheidungsträger aus Wirtschaft, Forschung und Politik von der breit angelegten Überwachung und Spionage durch „Prism“ und „Tempora“ betroffen sind. Bereits im Juli 2013 hatte der Sächsische Datenschutzbeauftragte den Ministerpräsidenten in einem Schreiben gebeten, auch auf sächsischer Ebene notwendige Konsequenzen zu ziehen. Dazu gehören unter anderem eine Untersuchung, inwieweit die von sächsischen Bürgern, Behörden und Unternehmen genutzten Übertragungswege und gespeicherten Daten derzeit gesichert sind und zukünftig geschützt werden können, die Förderung und Erprobung von technischen Maßnahmen zur Datensicherheit und Verschlüsselung, die Förderung lokaler Clouddienste, die Stärkung des Staatsbetriebes Sächsische Informatik Dienste (SID) und der für die Spionageabwehr zuständigen Abteilung des LfV Sachsen, die Intensivierung der Beratung staatlicher und privater Einrichtungen über Informationssicherheitserfordernisse sowie die Stärkung des Selbstdatenschutz-Gedankens, der 